\title{
Effects of tin caulis dendrobium polysaccharide on the prevention of non-alcoholic fatty liver disease in rats
}

\author{
Dingting $\mathrm{Xu}^{1}$, Hanyun Zhang ${ }^{1}$, Xiaoying Wang ${ }^{1 *}$
}

${ }^{1}$ Zhejiang University, School of Medicine, The Second Affiliated Hospital, Hangzhou, China.

\begin{abstract}
We aimed to investigate the effects and mechanisms of tin caulis dendrobium polysaccharide on body weight and non-alcoholic fatty liver disease in Sprague-Dawleg rats. The rats were fed a high-fat diet (HFD) with or without tin caulis dendrobium polysaccharide for eight weeks. Body weight, liver function tests, serum and liver lipids, liver morphology, and the expression of PPAR $\gamma$ and UCP2 were assessed. Rats fed HFD exhibited more obvious weight gain, increased serum, and hepatic lipids. But the more tin caulis dendrobium polysaccharide they fed, the lower increase in weight, serum, and hepatic lipids of rats were found. Furthermore, the expression of PPAR $\gamma$ and UCP2 decreased in rats fed tin caulis dendrobium polysaccharide compared with HFD-fed rats. Tin caulis dendrobium polysaccharide may downregulate the expression of $\operatorname{PPAR} \gamma$ and UCP2 in liver and protect against body weight gain and liver fat deposition.
\end{abstract}

Key Words: fatty liver, high-fat diet, intervention, mechanisms

\section{Introduction}

Non-alcoholic fatty liver disease (NAFLD) has been a disturbing clinical problem. In recent decades, the prevalence of NAFLD has significantly increased (Fan et al., 2005) and will replace viral hepatitis as the most common liver disease in China. Non-alcoholic fatty liver disease is closely related to obesity. Studies have found that fat metabolism in liver is associated with energy metabolism; many kinds of metabolites, hormones, cytokines, and neurotransmitters play an important part, such as tumor necrosis factor, leptin, and adiponectin. It is not only the result of dysbolism, but also the flare factor to normalize the liver fat metabolism. This course can change the activity of the above-mentioned regulators in other tissues and lead to insulin resistance, metabolic syndrome, tumors, and so on (Furukawa et al., 2004; Shiomi et al., 2013).

With the development of modern medical technology, many natural pigments from vegetables and fruits interest scientists and their non-toxicity and beneficial health effects attract people for prophylaxis or treatment, such as purified

Received: September 18, 2016

Accepted: March 12, 2017

*Corresponding author: 2503024@zju.edu.cn

http://dx.doi.org/10.1590/S1806-92902017000800004

How to cite: Xu, D.; Zhang, H. and Wang, X. 2017. Effects of tin caulis dendrobium polysaccharide on the prevention of non-alcoholic fatty liver disease in rats. Revista Brasileira de Zootecnia 46(8):652-656.

Copyright (C) 2017 Sociedade Brasileira de Zootecnia. This is an Open Access article distributed under the terms of the Creative Commons Attribution License (http://creativecommons.org/licenses/by/4.0/), which permits unrestricted use, distribution, and reproduction in any medium, provided the original work is properly cited. mulberry (Wu et al., 2013a), blueberry (Wu et al., 2013b), and resveratrol (Heeboll et al., 2015). Tin caulis dendrobium is widely used for antipyretic, immunomodulatory, and cardiovascular protection in traditional Chinese medicine, known as "Shihu" or "Fengdou". But whether tin caulis dendrobium can prevent the development of fatty liver disease is not clearly. In this study, we fed rats with tin caulis dendrobium polysaccharide and investigated its effect on the development of NAFLD.

\section{Material and Methods}

Ninety-five percent of dendrobium polysaccharide were obtained from Pro Tianhong Biotech Co., Ltd. (Hangzhou, P.R.China). Cholesterol, bile salts, egg yolk powder, edible lard, and the basal diet were purchased from Suyisi Biological Pharmaceutical Co., Ltd. (Jiangsu, P.R.China). Hematoxylin and eosin staining materials were purchased from Health Engineering Limited (Shanghai, P.R.China).

The experimental protocol was approved by the local Experimental Animal Ethics Committee (Case no. ZJU201308-1-10-072) and performed under the National Institutes of Health Guide for Care and Use of Laboratory Animals.

Seventy-two male Sprague-Dawleg (SD) rats weighing $105 \pm 5 \mathrm{~g}$ were purchased from Zhejiang Academy of Medical Science (certificate no. 22-2001001, Hangzhou, China).

All animals were maintained under standard conditions of temperature $\left(22 \pm 1^{\circ} \mathrm{C}\right)$ and light $(12 \mathrm{~h} \mathrm{light}$ and $12 \mathrm{~h}$ dark cycle) and fed standard laboratory chow and tap water 
ad libitum. All procedures were in strict accordance with the PR China legislation on use. After 1-week adaptation, all experimental rats $(n=72)$ were randomly divided into six groups and fed specific diets for eight weeks. According to the daily feed intake, the groups included: the control group fed low-fat diet (LFD, 10\% calories from fat); the control group fed LFD plus dendrobium polysaccharide (DEN, $500 \mathrm{mg}$ per $\mathrm{kg}$ of feed); the control group fed a high-fat diet (HFD, $45 \%$ calories from fat); DL - the test group fed HFD plus low dose (250 mg) of DEN per kg of feed); DM - the test group fed HFD plus medium dose $(500 \mathrm{mg})$ of DEN per kg of feed; and DH - the test group fed HFD plus high dose $(1000 \mathrm{mg})$ of DEN per $\mathrm{kg}$ of feed. Body weight and the feed intake were measured once a week.

At week 8, blood samples were collected. After standing at room temperature, blood samples were centrifuged at 3000 revolutions $/ \mathrm{min}$ for $15 \mathrm{~min}$. Then, the serum was isolated and stored at $-20{ }^{\circ} \mathrm{C}$. Concentrations of serum alanine aminotransferase (ALT), aspartate aminotransferase (AST), triglyceride (TG), and total cholesterol (TC) were determined by biochemical auto analyzers.

At week 8 , the rats were sacrificed by suffocation in nitrogen gas. After death, the abdominal cavity was opened and liver was isolated. Then liver was fixed with $4 \%$ paraformaldehyde for $2 \mathrm{~h}$. Liver was washed in PBS three times ( $5 \mathrm{~min}$ at every turn). Specimens were embedded in paraffin, then cut (4-7- $\mu \mathrm{m}$ thickness), stained with hematoxylin/eosin, and examined under light microscopy.

Liver tissue was snap-frozen in liquid nitrogen and total RNA was isolated and reverse transcribed with reverse transcriptase in the presence of random hexamers, according to manufacturer's instructions. Expression of PPAR $\gamma$ and UCP2 mRNA were analyzed using quantitative RT-PCR (Toyobo Revertra Ace qPCR RT kit, SYBR Green PCR kit, Thermo). The expression level was standardized to glyceraldehydephosphate dehydrogenase (GAPDH) of rats. The following primer sequences were used: PPAR $\gamma$ (forward): 5'-CGCTGATGCACTGCCTATGA-3'; PPAR $\gamma$ (reverse): 5'-AGAGGTCCACAGAGCTGATTC-3'; UCP2 (forward): 5'- CCGCATTGGCCTCTACGACTCT-3-3'; UCP2 (reverse): 5'-CCCCGAAGGCAGAAGTGAAGTG-3'.

Results were expressed as the means \pm SEM (standard error of means). The statistical evaluation of the differences were compared between the different groups by one-way ANOVA. All data were processed and analyzed by SPSS13.0 Data Editor (SPSS Inc., Chicago, IL, USA). The results in comparisons between groups were considered different if $\mathrm{P}<0.05$.

\section{Results}

All rats fed HFD added with dendrobium polysaccharide for eight weeks were healthy. Initial average body weight of rats were $104.8 \mathrm{~g}, 105.5 \mathrm{~g}$, and $106.7 \mathrm{~g}$, which showed no significant difference from HFD group. After eight weeks, rats fed only HFD gained greater body weight and the weight growth rate was $280 \%$ (Table 1). Intake of DEN prevented body weight increase for the HFD-fed rats. High dose of DEN had the most significant effect, the effect of medium dose was the second most significant $(\mathrm{P}<0.05)$ (Table 1).

Rats fed LFD showed lower lipid accumulation (Figure 1A), while rats fed HFD showed intense lipid accumulation in the liver (Figure 1C). In contrast, rats fed HFD added with DEN had significantly alleviating lipid accumulation (Figure 1D-F). Furthermore, the protective action strength of DEN on rats was in direct ratio to the dosages of DEN. Group of medium and high dose of DEN showed morphology change similar to LFD group.

Serum ALT, AST, TG, and TC levels elevated compared with other groups. Rats fed DEN showed that the levels of serum parameters decreased slightly compared with HFD group. High doses of DEN significantly reduced AST by $12.3 \%$, TC by $34.2 \%$, and TG by $38.8 \%(\mathrm{P}<0.05)$. Medium dose of DEN significantly reduced AST by $9.8 \%$, TC by $33 \%$, and TG by $38.9 \%(\mathrm{P}<0.05)$. Low dose of DEN showed no significant effect on serum parameters (Table 2).

Relative mRNA expression levels of PPAR $\gamma$ and UCP2 in the liver increased in rats fed HFD compared with those

Table 1 - Body weight of Sprague-Dawleg rats

\begin{tabular}{|c|c|c|c|c|c|c|c|c|c|}
\hline Group & Week 0 & Week 1 & Week 2 & Week 3 & Week 4 & Week 5 & Week 6 & Week 7 & Week 8 \\
\hline LFD & $106.2 \pm 6.9$ & $42.4 \pm 10.1$ & $86.6 \pm 14.9$ & $224.2 \pm 15.2 \mathrm{a}$ & $16.7 \mathrm{a}$ & $286.5 \pm 15.4 \mathrm{a}$ & $302.6 \pm 19.6 \mathrm{a}$ & $323.8 \pm 20.1 \mathrm{a}$ & 337.2 \\
\hline DEN & $107.3 \pm 6.5$ & $145.1 \pm 11.9$ & $182.5 \pm 15.7$ & $226.8 \pm 15.9 a$ & $261.9 \pm 17.1 \mathrm{a}$ & $282.9 \pm 14.8 \mathrm{a}$ & $306.5 \pm 18.9 a$ & $327.2 \pm 19.5 \mathrm{a}$ & $342.7 \pm 17.6 a$ \\
\hline HFD & $105.9 \pm 7.2$ & $156.8 \pm 12.6$ & $201.5 \pm 16.9$ & $251.6 \pm 17.2 b$ & $289.6 \pm 17.8 b$ & $328.8 \pm 17.4 \mathrm{c}$ & $354.3 \pm 21.2 \mathrm{c}$ & $382.9 \pm 22.6 \mathrm{c}$ & $399.4 \pm 17.9 b$ \\
\hline DL & $104.8 \pm 7.6$ & $154.3 \pm 13.8$ & $197.8 \pm 16.1$ & $242.5 \pm 19.3 \mathrm{ab}$ & $282.9 \pm 19.7 \mathrm{ab}$ & $317.6 \pm 18.9 b c$ & $349.6 \pm 28.3 b c$ & $371.3 \pm 27.5 b c$ & $385.6 \pm 18.2 b$ \\
\hline DM & $105.5 \pm 6.8$ & $147.5 \pm 12.7$ & $192.7 \pm 15.9$ & $233.8 \pm 17.9 \mathrm{ab}$ & $275.3 \pm 17.4 \mathrm{ab}$ & $302.4 \pm 22.5 \mathrm{ab}$ & $329.1 \pm 23.1 \mathrm{abc}$ & $343.8 \pm 24.3 \mathrm{ab}$ & $356.6 \pm 18.7 \mathrm{a}$ \\
\hline $\mathrm{DH}$ & $106.7 \pm 6.4$ & $151.4 \pm 13.2$ & $195.9 \pm 16.4$ & $235.2 \pm 17.6 \mathrm{ab}$ & $272.8 \pm 18.2 \mathrm{ab}$ & $298.9 \pm 19.7 \mathrm{ab}$ & $325.7 \pm 21.7 \mathrm{ab}$ & $346.6 \pm 24.8 \mathrm{ab}$ & $354.8 \pm 20.1 \mathrm{a}$ \\
\hline
\end{tabular}

LFD - rats fed low-fat diet; DEN - rats fed LFD plus dendrobium polysaccharide (DEN, 500 mg per kg feed); HFD - rats fed high-fat diet; DL - rats fed HFD plus low dose $(250 \mathrm{mg})$ of DEN per kg of feed; DM - rats fed HFD plus medium dose $(500 \mathrm{mg})$ of DEN per kg of feed; DH - rats fed HFD plus high dose (1000 mg) of DEN per kg of feed. Different letters indicate a significant difference between columns $(\mathrm{P}<0.05)$. 
fed LFD. Peroxisome proliferator-activated receptorgamma and UCP2 mRNA expression showed a significant decrease in rats fed HFD added with high dose and medium dose of DEN compared with HFD group, while similar to LFD group (Figure 2).

\section{Discussion}

With the development of modern medical technology, extraction of natural function factors from plants have interested scientists. As reported, dendrobium polysaccharide extracted from tin caulis dendrobium have biological effectiveness, such as cardiovascular protection and tumor inhibition (Gordon and Roedig-Penman, 1998).
However, it is unknown whether dendrobium polysaccharide can protect liver from non-alcoholic fatty liver disease. In this study, we fed rats a high-fat diet or low-fat diet, added with or without dendrobium polysaccharide, and observed the effect of dendrobium polysaccharide on obese rats.

Sprague-Dawleg rats are particularly sensitive to diet-induced obesity and are widely used in studies of metabolic disease, which is associated with obesity. As expected, in our study, the rats fed HFD had significant increase in body weight and serum analysis showed that glucose and lipid metabolism were affected, as well as the elevation of alanine aminotransferase and aspartate aminotransferase. These findings were in agreement with previous reports (Gordon and Roedig-Penman, 1998).

Table 2 - Serum parameters of Sprague-Dawleg rats

\begin{tabular}{lcccc}
\hline Group & ALT $(\mathrm{U} / \mathrm{L})$ & AST $(\mathrm{U} / \mathrm{L})$ & TC $(\mathrm{mmol} / \mathrm{L})$ & TG $(\mathrm{mmol} / \mathrm{L})$ \\
\hline LFD & $62.15 \pm 12.53 \mathrm{a}$ & $113.62 \pm 26.85 \mathrm{a}$ & $1.91 \pm 0.21 \mathrm{a}$ & $0.51 \pm 0.19 \mathrm{a}$ \\
DEN & $65.82 \pm 13.15 \mathrm{a}$ & $116.25 \pm 28.17 \mathrm{ab}$ & $1.95 \pm 0.23 \mathrm{a}$ & $3.18 \pm 0.27 \mathrm{c}$ \\
HFD & $91.26 \pm 14.53 \mathrm{c}$ & $162.18 \pm 31.26 \mathrm{c}$ & $2.83 \pm 0.26 \mathrm{~b}$ & $1.21 \pm 0.43 \mathrm{c}$ \\
DL & $86.93 \pm 13.29 \mathrm{bc}$ & $155.42 \pm 29.58 \mathrm{bc}$ & $2.13 \pm 0.19 \mathrm{a}$ & $1.13 \pm 0.46 \mathrm{~b}$ \\
DM & $73.52 \pm 13.17 \mathrm{ab}$ & $146.17 \pm 25.62 \mathrm{ab}$ & $0.71 \pm 0.22 \mathrm{ab}$ & $0.74 \pm 0.25 \mathrm{ab}$ \\
DH & $69.83 \pm 13.21 \mathrm{a}$ & $142.84 \pm 23.58 \mathrm{ab}$ & $2.09 \pm 0.23 \mathrm{a}$ & \\
\hline
\end{tabular}

ALT - alanine aminotransferase; AST - aspartate aminotransferase; TG - triglyceride; TC - total cholesterol; LFD - rats fed low-fat diet; DEN - rats fed LFD plus dendrobium polysaccharide (DEN, $500 \mathrm{mg}$ per kg feed); HFD - rats fed high-fat diet; DL - rats fed HFD plus low dose (250 mg) of DEN per kg of feed; DM - rats fed HFD plus medium dose $(500 \mathrm{mg})$ of DEN per $\mathrm{kg}$ of feed; DH - rats fed HFD plus high dose $(1000 \mathrm{mg})$ of DEN per $\mathrm{kg}$ of feed.

Different letters indicate a significant difference among columns $(\mathrm{P}<0.05)$.
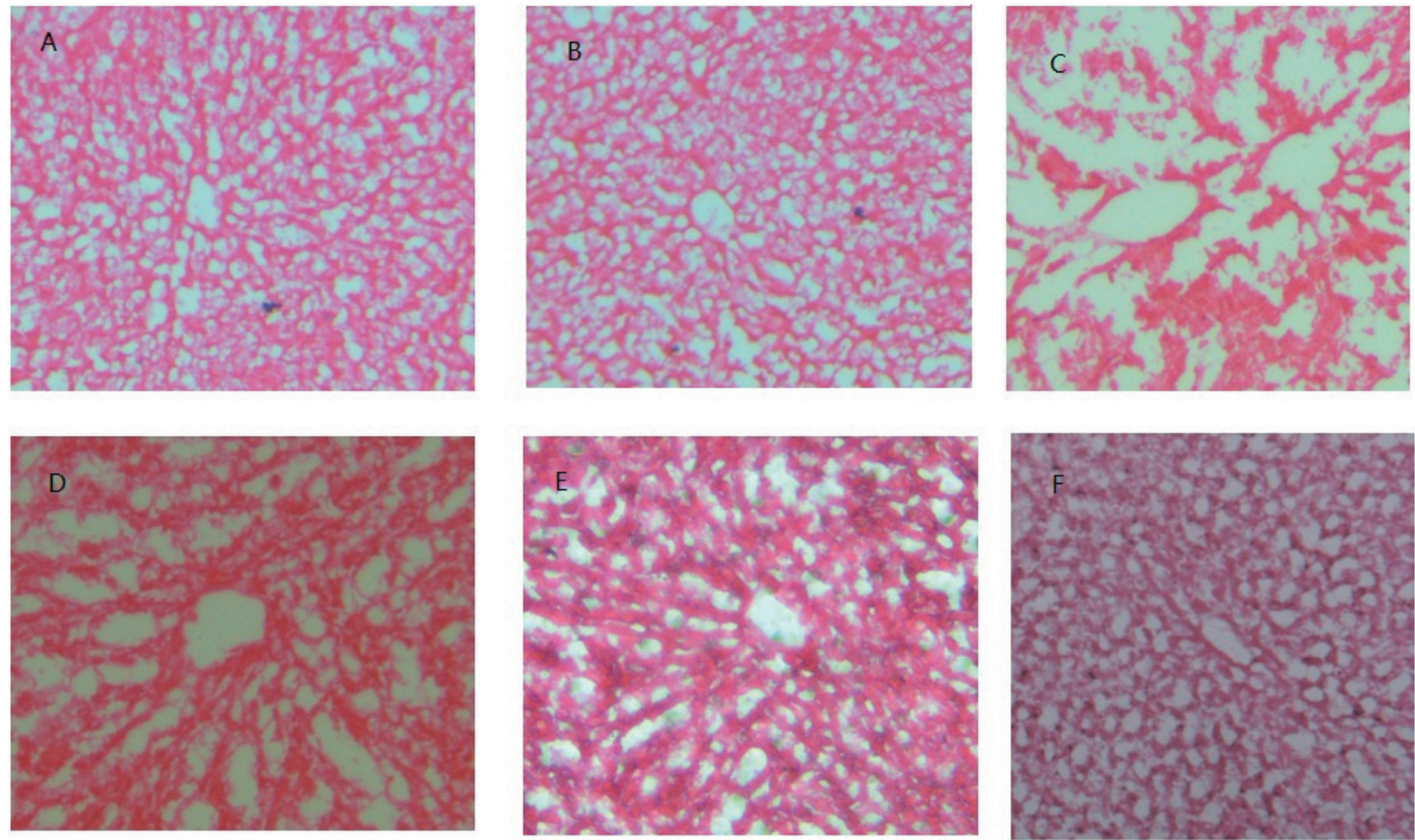

A: rats fed low-fat diet; B: rats fed low-fed diet plus dendrobium polysaccharide (500 mg per kg of feed); C: rats fed high-fat diet; D: rats fed high-fat diet plus low dose (250 mg) of dendrobium polysaccharide per kg of feed; E: rats fed high-fat diet plus medium dose $(500 \mathrm{mg})$ of dendrobium polysaccharide per kg of feed; F: rats fed high-fat diet plus high dose $(1000 \mathrm{mg})$ of dendrobium polysaccharide per $\mathrm{kg}$ of feed.

Figure 1 - Morphology changes in liver of Sprague-Dawleg rats. 


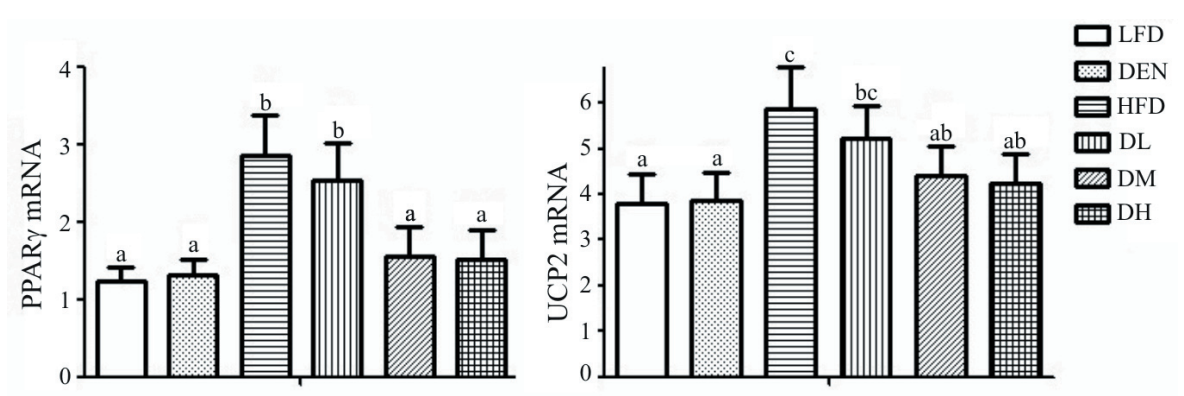

LFD - rats fed low-fat diet; DEN - rats fed LFD plus dendrobium polysaccharide (DEN, 500 mg per kg feed); HFD - rats fed high-fat diet; DL - rats fed HFD plus low dose $(250 \mathrm{mg})$ of DEN per kg of feed; DM - rats fed HFD plus medium dose $(500 \mathrm{mg})$ of DEN per kg of feed; DH - rats fed HFD plus high dose (1000 mg) of DEN per kg of feed. Bars with different letters differ significantly $(\mathrm{P}<0.05)$

Figure 2 - Relative mRNA expression in different groups by quantitative real-time PCR.

On the other hand, diet added with different doses of dendrobium polysaccharide can reduce the increase of ALT, AST, TG, and TC levels in obese rats. The liver and adipose tissue analysis showed that dendrobium polysaccharide can also improve fat tissue hypertrophy and excessive fatty deposition in liver. Dendrobium polysaccharide showed the effect on preventing body weight and there was a positive correlation between the action strength and the dose of DEN. This result showed a dose-dependent manner (McClung et al., 2004). However, the mechanism of this increase tendency with the higher dose of DEN is still unclear.

Peroxisome proliferator-activated receptors (PPAR) belong to ligand-activated transcription factors, a member of the metabolic nuclear receptor superfamily that regulate the activation of a variety of important target genes, and can be divided into three subtypes named PPAR $\alpha$, PPAR $\beta$, and PPAR $\gamma$. The PPAR $\gamma$-mediated target genes are closely associated with adipocyte differentiation, obesity, and insulin resistance (Vitale et al., 2016). During differentiation of preadipocytes and adipocytes, PPAR $\gamma$ is an important positive regulatory factor (Huan et al., 2003; Qi et al., 2015). On the other hand, the main part of body heat production is in the mitochondria and the quantity of heat depends on uncoupling protein (UCP) of mitochondria. In mammals, there are five types of uncoupling protein: UCP1, UCP2, UCP3, UCP4, and UCP5. The gene of UCP2 is located on human chromosome 11 and murine chromosome 7, associated with obesity (Zhang et al., 2014). Our study showed that the expression of PPAR $\gamma$ and UCP2 in liver fat tissue were significantly lower in obesity-resistant rats than in obesity-prone rats, which may be related to reducing steatosis, insulin resistance, and leptin resistance. It is a possible molecular mechanism of diet-induced obesity resistance (Steppan et al., 2001; Qi et al., 2015).

\section{Conclusions}

Dendrobium polysaccharide could significantly protect liver from fat deposition. Dendrobium polysaccharide may reduce the expression of PPAR $\gamma$ and UCP2, then improve lipid peroxidation and the development of obesity and fatty liver. Our results can provide the scientific base for intervention of nonalcoholic fatty liver by Dendrobium diet. Although the signaling pathway of how dendrobium polysaccharide effects on obesity is still unknown, dendrobium polysaccharide could be used to improve the development of non-alcoholic fatty liver disease in clinical.

\section{Acknowledgments}

This work was supported by Natural Science Foundation of Zhejiang Province, China (grant number LY15H160040).

\section{References}

Fan, J. G.; Zhu, J.; Li, X. J.; Chen, L.; Li, L.; Dai, F.; Li, F. and Chen, S. Y. 2005. Prevalence of and risk factors for fatty liver in a general population of Shanghai, China. Journal of Hepatology 43:508-514

Furukawa, S.; Fujita, T.; Shimabukuro, M.; Iwaki, M.; Yamada, Y.; Nakajima, Y.; Nakayama, O.; Makishima, M.; Matsuda, M. and Shimomura, I. 2004. Increased oxidative stress in obesity and its impact on metabolic syndrome. Journal of Clinical Investigation 114:1752-1761.

Gordon, M. H. and Roedig-Penman, A. 1998. Antioxidant activity of quercetin and myricetin in liposomes. Chemistry and Physics of Lipids 97:79-85.

Heeboll, S.; Thomsen, K. L.; Clouston, A.; Sundelin, E. I.; Radko, Y.; Christensen, L. P.; Ramezani-Moghadam, M.; Kreutzfeldt, M.; Pedersen, S. B.; Jessen, N.; Hebbard, L. and George, J. and Gronbaek, H. 2015. Effect of resveratrol on experimental nonalcoholic steatohepatitis. Pharmacological Research 95-96:34-41. 
Huan, J. N.; Li, J.; Han, Y.; Chen, K.; Wu, N. and Zhao, A. Z. 2003. Adipocyte-selective reduction of the leptin receptors induced by antisense RNA leads to increased adiposity, dyslipidemia, and insulin resistance. Journal of Biological Chemistry 278:4563845650.

McClung, J. P.; Roneker, C. A.; Mu, W.; Lisk, D. J.; Langlais, P.; Liu, F. and Lei, X. G. 2004. Development of insulin resistance and obesity in mice overexpressing cellular glutathione peroxidase. Proceedings of the National Academy of Sciences of United States of America 101:8852-8857

Qi, Z. G.; Zhao, X.; Zhong, W. and Xie, M. L. 2015. Osthole improves glucose and lipid metabolism via modulation of PPARalpha/ gamma-mediated target gene expression in liver, adipose tissue, and skeletal muscle in fatty liver rats. Pharmaceutical Biology 54:882-888.

Shiomi, K.; Kuriyama, I.; Yoshida, H. and Mizushina, Y. 2013. Inhibitory effects of myricetin on mammalian DNA polymerase, topoisomerase and human cancer cell proliferation. Food Chemistry 139:910-918.
Steppan, C. M.; Bailey, S. T.; Bhat, S.; Brown, E. J.; Banerjee, R. R.; Wright, C. M.; Patel, H. R.; Ahima, R. S. and Lazar, M. A. 2001. The hormone resistin links obesity to diabetes. Nature 409:307-312.

Vitale, S. G.; Lagana, A. S.; Nigro, A.; La Rosa, V. L.; Rossetti, P.; Rapisarda, A. M.; La Vignera, S.; Condorelli, R. A.; Corrado, F.; Buscema, M. and D'Anna, R. 2016. Peroxisome proliferatoractivated receptor modulation during metabolic diseases and cancers: Master and minions. PPAR Research 2016:6517313.

Wu, T.; Qi, X.; Liu, Y.; Guo, J.; Zhu, R.; Chen, W.; Zheng, X. and Yu, T. 2013a. Dietary supplementation with purified mulberry (Morus australis Poir) anthocyanins suppresses body weight gain in high-fat diet fed C57BL/6 mice. Food Chemistry 141:482-487.

Wu, T.; Tang, Q.; Gao, Z.; Yu, Z.; Song, H.; Zheng, X. and Chen, W. 2013b. Blueberry and mulberry juice prevent obesity development in C57BL/6 mice. PLoS One 8:e77585.

Zhang, M.; Wang, M. and Zhao, Z. T. 2014. Uncoupling protein 2 gene polymorphisms in association with overweight and obesity susceptibility: A meta-analysis. Meta Gene 2:143-159. 